Introduction In order to prevent neurodegenerative diseases such as chronic solvent encephalopathy (CSE), it is important to elucidate the underlying mechanism. Cells are able to respond to occupational stressors by turning on or off specific genes mediated through epigenetic alterations. Consequently, epigenetic mechanisms can play an important role in mediating the effects of solvent exposure on the development of neurobehavioral disorders.

Methods We have set-up several studies in a translational design using cell lines, mice and humans exposed to solvents and CSE patients. DNA methylation was measured with liquid chromatography coupled with tandem mass spectrometry for the assessment of 2'-deoxycytidine, 5-methyl-2'-deoxycytidine, 5 -hydroxymethyl-2'-deoxycytidine, 5-formyl-2'-deoxycytidine, 5 -carboxy-2'-deoxycytidine and pyrosequencing.

Results Global DNA methylation and hydroxymethylation was mostly negatively associated with solvent exposure in both in vitro as in vivo studies. DNMT1 CpG1 gene-specific methylation levels were significantly higher in CSE patients as compared to controls, associations that persisted after adjustment for age, gender, alcohol abuse, smoking, level of education and the use of psychotropic medication $(p=0.004$ and $\mathrm{p}=0.017$, respectively). In addition, in the group of CSE patients there was a positive correlation between the duration of solvent exposure and the percentage of BDNF CpG1 methylation ( $r s=0.374, p=0.017)$, which was maintained after adjustment for age and smoking $(\mathrm{p}<0.001)$.

Conclusion The study of epigenetic alterations gives us the possibility to study the immediate consequences of exposure and make projections for disease development. These epigenetic marks can be used to develop a set of biomarkers and used in the follow-up of humans.

\section{3d OCCUPATIONAL EXPOSURE TO SOLVENTS AND RISK OF PARKINSON DISEASE IN FINLAND}

Brad A Racette. Washington University School of Medicine, St. Louis, USA, and University of the Witwatersrand School of Public Health, Johannesburg, South Africa

\subsection{6/oemed-2018-ICOHabstracts.810}

Background Prior epidemiologic and animal studies indicate that occupational exposure to solvents, particularly trichloroethylene, may increase risk of Parkinson disease (PD).

Methods We constructed a population-based case-control study of incident PD in Finland using a medication database maintained by the Social Insurance Institution of Finland, along with the Population Information System, which includes census records for all Finnish residents. PD cases were diagnosed (first approved to receive PD medications) between 19952014. We randomly selected controls from the underlying population while matching on diagnosis year, birth year (1930-1950), and sex. Individual occupational census data from 1990, allowing for a minimum of five years of exposure lagging, were available for 11,757 PD cases and 23236 controls. These data were linked to the Finnish Job Exposure Matrix (FINJEM) to identify potential for occupational exposure to four classes of solvents. We estimated PD-solvent odds ratios (ORs) and 95\% confidence intervals (CIs) using unconditional logistic regression, while adjusting for matching variables and socioeconomic status as a proxy for tobacco smoking.

Results Cases and controls were age 45-89 years at diagnosis/ reference and age 40-60 years at occupational exposure assessment. There was no evidence that PD was associated with occupational exposure to solvents overall (19\% cases and $20 \%$ controls), or to aromatic hydrocarbon solvents in particular, with ORs very close to null. In contrast, there was a modestly higher PD risk in relation to exposure to chlorinated hydrocarbon solvents, most notably trichloroethylene $(\mathrm{OR}=1.11,95 \% \mathrm{CI}: 0.98$ to 1.26$)$. Occupation as a mechanic $(\mathrm{OR}=1.20,95 \% \mathrm{CI}: 0.97$ to 1.49$)$ or as an electronic or telecommunications worker $(\mathrm{OR}=1.65,95 \% \mathrm{CI}$ : 1.07 to 2.54$)$ contributed to this association.

Conclusion Consistent with prior literature, occupational exposure to tricholoethylene may at least modestly increase risk of PD.

\section{ENVIRONMENTAL INTOLERANCE - A CONTINUUM FROM ANNOYANCE TO SEVERE ILLNESS}

${ }^{1}$ Markku A Sainio*, ${ }^{2,3}$ Martin Tondel*. ${ }^{1}$ Work Environment, Finnish Institute of Occupational Health, Helsinki, Finland; ${ }^{2}$ Occupational and Environmental Medicine, Uppsala University Hospital, Uppsala, Sweden; ${ }^{3}$ Occupational and Environmental Medicine, Department of Medical Sciences, Uppsala University, Uppsala, Sweden

\subsection{6/oemed-2018-ICOHabstracts.811}

Aim of the special session Intolerance may develop to various everyday environmental exposures at levels that are well below those known to cause adverse health effects. Reactions initiate typically from odorous substances, but also from nonperceivable factors considered harmful to health, e.g. electrical devices. Reactions range from unpleasant sensations and annoyance to multi-organ symptoms, severe disability, and major restrictions in daily life and work. Symptoms often lead to exposure assessments at work and may result in excessive actions to eliminate minor exposures. In environmental intolerance, occupational health care is the front line actor in primary and secondary prevention, and support of recovery.

Markku Sainio ${ }^{1}$, Anna-Sara Claeson ${ }^{2}$, Christoph van Thriel $^{3}$, Kenichi Azuma ${ }^{4}$, Lena Hillert ${ }^{5}$, Martin Tondel ${ }^{6,7}$

${ }^{1}$ Work Environment, Finnish Institute of Occupational Health, Helsinki, Finland

${ }^{2}$ Department of Psychology, Umeå University, Sweden

${ }^{3}$ Leibniz Research Centre for Working Environment and Human Factors, TU Dortmund, Dortmund, Germany

${ }^{4}$ Kindai University Faculty of Medicine, Osakasayama, Japan

${ }^{5}$ Institute of Environmental Medicine, Karolinska Institutet, Stockholm, Sweden

${ }^{6}$ Occupational and Environmental Medicine, Uppsala University Hospital, Uppsala, Sweden

${ }^{7}$ Occupational and Environmental Medicine, Department of Medical Sciences, Uppsala University, Uppsala, Sweden

\section{6a ENVIRONMENTAL INTOLERANCE - DEFINITIONS AND RELEVANCE TO OCCUPATIONAL HEALTH}

M Sainio, K Karvala, A Vuokko. Finnish Institute of Occupational Health, Work Environment, Helsinki, Finland

10.1136/oemed-2018-ICOHabstracts.812

Number of people attribute symptoms to everyday environmental exposures at levels that are well below those known to cause adverse health effects and that are tolerated by the majority. Intolerance may develop to various environmental 